\title{
Aiding adherence: five approaches to following prescriptions
}

Poor adherence to medications costs the US as much as $\$ 290$ billion a year in increased healthcare costs, according to the not-for-profit New England Healthcare Institute. That cost arises from roughly half of all patients failing to take medications as prescribed ( $N$. Engl. $J$. Med. doi:10.1056/nejmp1002305, 2010). Beyond cost and failure to properly treat a medical condition, it's been known for some time that the failure to follow prescribed medical regimens can also trigger resistance among a range of microbes.

The lack of patient adherence arises from cost, inconvenience, forgetfulness and other factors. So experts have called for innovation in this area. As Lars Osterberg, chief of general internal medicine at the VA Palo Alto Health Care System in California, says, "we must coordinate a number of tools to improve the adherence for individuals." Here are a handful of approaches to help make that happen.

\section{Forget-proof top}

Many people think of caps on pharmaceutical bottles as deterring access to pills, because they are often childproof, but GlowCaps, from Vitality in Cambridge, Massachusetts, encourage patients to open bottles. A patient replaces an ordinary medication-bottle cap with one that includes a wireless chip. This specially designed chip continuously communicates with a reminder device, which gets plugged in near where the patient keeps his or her medications. Then, the patient makes a telephone call to GlowCaps' customer service to provide information about when the medication should be taken.

When the time comes to take the medicine, the cap flashes. If the cap doesn't get opened, the system plays a little tune. If that fails, the system calls the patient with an automated voice message.

This company also provides an option in certain circumstances for a family member to receive a weekly report of the patient's adherence to the medicine. That information can also be shared with the patient's physician. The system can even call in a refill before the prescription runs out. Unpublished company tests claim that GlowCaps drive an average adherence of $86 \%$.

\section{Thinking outside the box}

Today's pillboxes provide more possibilities. The Maya system from MedMinder in Newton, Massachusetts, for instance, consists of 28 compartments, arranged as four per day of the week, and it can be programmed to light up or beep when a patient should take the pills in a specific compartment. On-board electronics and an embedded cellular modem — no need to connect to a phone line or the Internet-

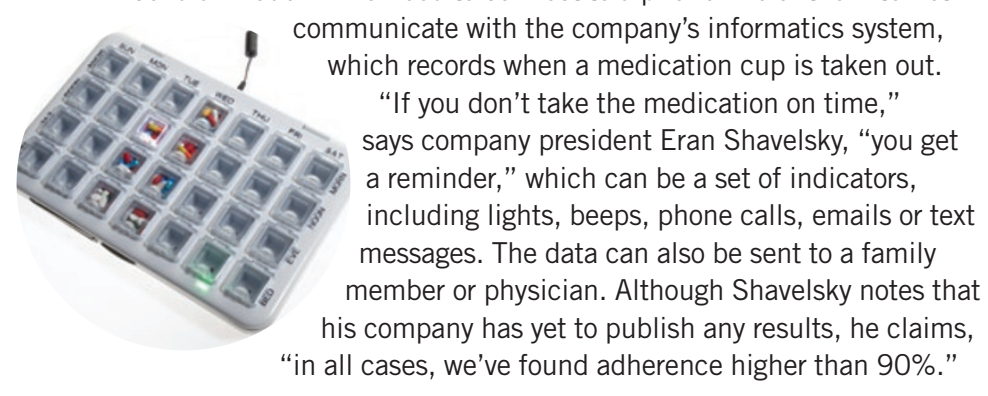

\section{Roll with it}

Adherence gets more difficult when someone takes multiple medications, with different combinations at different times. "If someone is taking ten meds, how do you make it convenient for them?" Osterberg asks. One way, he points out, is through better packaging, such as plastic tubes pinched into packets containing multiple pills.

The company Talyst in Bellevue, Washington has designed a machine that combines various pills into plastic packages according to each patient's regimen. Imagine a roll of these packages where each one includes the pills that a person is supposed to take at a specific time. "You can conveniently tear off the package, which includes a time stamped on it for when you take it and what's in the package," explains Osterberg, who is not involved with Talyst.

\section{Swallow the evidence}

To truly measure adherence requires recording when a pill is actually taken, which is possible with the Raisin System from Proteus Biomedical in Redwood City, California. This technologynamed after a fruit in the company's hopes that it will gain the success of Apple and Blackberry-depends on a one-millimeter silicon chip, which is coated on opposite sides with minerals that act like a battery when wet. This grain is attached to a pill, and it is turned on when the pill is consumed (moisture activates the battery). Then, a circuit inside the grain signals the serial number of the pill, which can be captured by a patient-worn patch.

Unlike a radio-frequency identification (RFID) chip, which transmits a signal through the air, the Raisin relies on the conductive characteristics of tissues to carry the signal to the receiver, so the chip only has to generate 100 microwatts of power. The patch can collect data, such as when a particular pill was taken, as well as biometric data. According to George Savage, chief medical officer at Proteus, this technology detected that a pill was taken in $97-98 \%$ of the more than 8,000 test consumptions, and the results have been presented at a variety of conferences. Later this year, Savage expects the Raisin to get a European CE mark, or Conformité Européenne, that indicates safety approval in the EU and to be approved by the US Food and Drug Administration by 2012.

\section{Getting personal}

Given the breadth of drivers behind poor adherence, it might take multiple approaches to remedy this problem. Using such a tactic, MEMOTEXT in Toronto combines a range of techniques into one product. A patient or his or her family or physician sets up a medication schedule through MEMOTEXT, which creates a tailored intervention for a him or her. These might include mailed treatment information and reminders and support through text messages and telephone calls. The patient's attitudes, disease literacy and behavior are assessed, and the results are combined with proprietary behavioral analysis to modify the intervention on an ongoing basis.

"For patients of an older demographic," explains company founder Amos Adler, "the intervention will more likely skew toward interventions that require less of a learning curve, such as touchtone telephone or natural language speech recognition. We use the most appropriate technology for the patient population and the condition." This technology is being tested in an ongoing study at Johns Hopkins University in Baltimore. Although the final results will not be available for about eight months, Adler says, "the preliminary findings are very positive."

Mike May, Houston 